Relations industrielles

Industrial Relations

\title{
Le travail en chantier par Marcelle Duc, Toulouse : Octares Éditions, 2002, 202 p., ISBN 2-906769-87-8.
}

\section{Jean Charest}

Volume 59, numéro 3, été 2004

URI : https://id.erudit.org/iderudit/010932ar

DOI : https://doi.org/10.7202/010932ar

Aller au sommaire du numéro

Éditeur(s)

Département des relations industrielles de l'Université Laval

ISSN

0034-379X (imprimé)

1703-8138 (numérique)

Découvrir la revue

Citer ce compte rendu

Charest, J. (2004). Compte rendu de [Le travail en chantier par Marcelle DUC, Toulouse : Octares Éditions, 2002, 202 p., ISBN 2-906769-87-8.] Relations

industrielles / Industrial Relations, 59(3), 626-628.

https://doi.org/10.7202/010932ar

Tous droits réservés @ C Département des relations industrielles de l'Université Laval, 2004
Ce document est protégé par la loi sur le droit d'auteur. L'utilisation des services d'Érudit (y compris la reproduction) est assujettie à sa politique d'utilisation que vous pouvez consulter en ligne.

https://apropos.erudit.org/fr/usagers/politique-dutilisation/ 
history was becoming more popular, dynamic and focused on social movements and organization.

However, according to van Voss and van der Linden, the 1980s - the fifth stage - changed that situation. Labour history fell out of favour with academics. Part of the reason for this was the ascendance of the discipline of women's studies because it tended to segregate the history of women from the history of men. Though it is pointed out that women's history is about all of mankind, not just half of it (p. 16), many academics saw the split between men's and women's history as encompassing more than just a division of historical facts. There are the issues of gendered language, private and public space, paid work, domestic work versus work outside the home - these issues remain at the root of the schism. Another reason for the shift away from labour history is that other identities such as religion or ethnic minorities were involved and had to be 'counted'.

Mike Savage's chapter, "Class and Labour History" goes beyond a Marxist perspective on labour history. Savage refuses to 'reduce' political and industrial conflict to class elements, instead he flags a new dimension - that working class life is characteristically built around insecurity, which means labour history should be looked at in "the contexts in which workers' lives are lived" (p. 69).

In the chapter "Gender in Labour History," Eileen Yeo outlines how gender is featured in class identity. She writes about the myth and reality of masculinity from the factory floor to the union hall and women's struggle to escape life in the "private sphere" of the 19th century. My interest in human resource management was sparked when Yeo referred to middle class women's role in industry prior to World War I. She writes that social work, as a profession, got its start when women worked as 'welfare officers' who watched over women workers in heavy industry. In that context, women got caught between the professional/managerial class and the working class. We know that twenty or thirty years earlier, it was mainly men who worked as 'welfare officers' and formed the first ranks of 'personnel officers'.

The book ends in a kind of puzzle. Does the exploration of social class widen or narrow the discussion of labour history? In the final chapter "Two Labour Histories of One?" Alice Kessler-Harris comes down somewhere in the middle. She opts for an expanded view of class which includes citizenship, gender, political rights and identity. But at the same time she tips her hat to the economic-focused view which was enunciated by the American leader Samuel Gompers who in 1898 told Congress, "a declaration of political liberty which does not involve an opportunity for economic independence is a delusion." (p. 147). Kessler-Harris says that labour history should be interpreted through the workers' relations to production and to consumption because there is more complexity to working class history than seeing it as merely class-based.

Judy HaIven

Saint Mary's University

\section{Le travail en chantier}

par Marcelle Duc, Toulouse : Octares Éditions, 2002, 202 p., ISBN 2-906769-87-8.

Derrière ce titre «jeu de mots » bien pensé se révèle un ouvrage fort intéressant sur l'organisation du travail, vue sous l'angle de la coopération, dans l'industrie 
du bâtiment (construction) en France. Le titre nous renvoie aussi à une analyse convaincante qui rappelle que l'étude du travail d'un point de vue sociologique n'est jamais achevée. Comme il se doit, expliquons brièvement la structure de l'ouvrage avant de le commenter davantage. On y retrouve quatre chapitres. Le premier est une analyse de la notion de coopération dans le travail telle que comprise et véhiculée de diverses manières dans les études sociologiques du travail. Le deuxième chapitre porte sur la description de l'organisation du travail dans l'industrie du bâtiment, notamment dans une perspective historique qui revient sur la période plus taylorienne de l'industrie et sur la période dite de redécouverte de la coopération que l'auteure situe aux années 70 et suivantes. Le troisième chapitre propose une lecture fine (au sens micro mais aussi quant à ses qualités) de l'activité de travail sur un chantier à partir des observations réalisées par la chercheure. Enfin, le quatrième et dernier chapitre porte sur l'activité d'encadrement et de gestion des hommes de chantier (supervision du travail), encore là à partir du travail d'observation de la chercheure. Une conclusion générale vient synthétiser les parcours multiples de l'ouvrage dont nous allons faire état maintenant.

L'intérêt de cet ouvrage est triple et celui-ci devrait convaincre, au moins dans une de ses facettes (sinon la totalité), les chercheurs qui s'intéressent à l'analyse du travail de s'y arrêter. D'abord, l'auteure nous propose une analyse très intéressante du traitement de la notion de coopération dans le travail par divers chercheurs, tel que mentionné plus haut. Des travaux précurseurs et limitatifs de cette notion (chez Marx), en passant par des considérations plus sociales relatives à l'activité de travail et à la coopération ouvrière (chez Friedmann et Touraine par exemple), l'auteure achève ce parcours dans le courant de la régulation sociale (qu'elle illustre surtout par les travaux de de Terssac). Elle en dégage une synthèse générale qui la conduit à l'idée de l'importance d'avoir une approche d'ouverture analytique et méthodologique à l'égard de l'objet qu'est le travail (et la coopération) notamment sous l'angle de la pluridisciplinarité en sciences sociales, approche qualifiée d'ergologique. Nous soulignons ici qu'une telle conclusion n'apparaît pas des plus surprenantes (ni novatrices sinon dans les termes) eu égard à la tradition des relations industrielles (comme champ d'étude) qui malheureusement est complètement absente dans l'analyse proposée. Le débat, et c'est là une limite du moins du point de vue du chercheur en relations industrielles mais aussi probablement du chercheur non français, demeure ici essentiellement interne à la sociologie française du travail.

Le deuxième intérêt de l'ouvrage est de nous introduire concrètement dans le milieu de la construction (du bâtiment) comme terrain privilégié par l'auteure pour y analyser le concept de coopération. Pourquoi cet intérêt? D'abord probablement parce qu'à la différence de beaucoup d'autres secteurs, celui-ci n'est pas le lieu habituel de l'analyse du travail en sciences sociales. On y apprendra donc beaucoup sur les périodes tantôt plus tayloriennes et tantôt plus coopératives de la conception du travail mais aussi sur la réalité quotidienne de l'exécution des activités, du travail de planification, de l'encadrement, le tout sous l'angle des rapports sociaux qui transcendent, voire dominent, une activité de travail qui en apparence est essentiellement technique, découpée, divisée en métiers indépendants, et vue souvent comme étant figée dans le temps depuis des années. Or, comme le souligne un des acteurs de l'industrie interviewés par la chercheure : «Dans ce métier on ne peut travailler qu'en équipe. C'est un axiome. Le Bâtiment c'est l'équipe» (p. 77). Et c'est là que s'appuie avec force selon nous le concept soutenu par 
l'auteure, soit «l'organisation du travail à prescription floue». Essentiellement, celui-ci vise à décrire une réalité du travail qui demande beaucoup de minutie dans la planification ex ante des activités de construction sans pouvoir en même temps être exécutées intégralement telles que planifiées, qui exige une coordination complexe de travaux réalisés par une multitude de métiers cloisonnés sur le plan professionnel, qui suppose une nécessaire anticipation de ce qui suivra le travail du moment, le tout mettant ainsi en relief l'importance des rapports entre tous les intervenants (qu'ils soient gestionnaires, superviseurs ou ouvriers). Ces rapports sont faits de communication, d'échange d'informations techniques, de discussion sur l'organisation du travail, et ils visent à pallier à l'impossibilité de tout prévoir en termes de déroulement des activités dans cette industrie. Des problèmes techniques surviennent sans cesse dans cet univers de production complexe (non 'standardisable' dans sa globalité par rapport à la production manufacturière de plusieurs biens) mais les solutions résident d'abord dans la qualité des rapports sociaux qu'entretiennent entre eux les divers intervenants. De là s'exprime l'incontournable lien entre les individus et le collectif dans cette industrie.
Le troisième intérêt de l'ouvrage réside dans ses aspects méthodologiques. La transparence de la démarche utilisée par la chercheure (faite d'observation, d'entrevues et de restitution - validation) et l'utilisation appropriée des citations en appui rappelle, d'une part, l'intérêt et la pertinence de la recherche dite qualitative en sciences sociales notamment pour saisir une réalité des plus complexes comme celle de l'analyse du travail et, d'autre part, indique bien les exigences méthodologiques d'une telle démarche. À cet égard, l'auteure ne nous laisse pas sous l'impression de forcer un cadre analytique sur une réalité qui pourrait échapper au lecteur. La démonstration est convaincante même si on peut parfois s'interroger sur certaines absences lors de la description des rapports de travail entre les divers intervenants. Pour notre part, il apparaît que les notions de conflictualité ou de tension dans ces rapports décrits par la chercheure sont trop peu explorées bien qu'elles soient une composante effective de la réalité du travail (dans ce secteur comme ailleurs) et bien qu'elles soient reliées conceptuellement à la notion de coopération.

Jean Charest

Université de Montréal

\section{Réfléchir la compétence : approches sociologiques, juridiques, économiques d'une pratique gestionnaire}

sous la direction de Arnaud Dupray, Christophe Guitton et Sylvie MonchATRE, Toulouse : Octares Éditions, 2003, 272 p., ISBN 2-90676997-5.

Cet ouvrage collectif rassemble les contributions produites à l'occasion d'un séminaire pluridisciplinaire tenu par le Centre d'études et de recherches sur les qualifications (Céreq) en France. L'objectif de ce séminaire était de «réfléchir la compétence» sous trois regards différents et complémentaires, soit ceux de la sociologie, du droit et de l'économie. Ce livre n'aborde pas la compétence comme un objet sans précédent, radicalement nouveau. Au contraire, il tend plutôt à banaliser ce concept en se demandant comment la compétence peut être considérée comme un objet ordinaire de la sociologie, du droit, de l'économie. Après une introduction générale, le contenu est divisé en trois parties distinctes regroupant, par discipline, les textes produits pour le séminaire du Céreq. 